\title{
Impact of imatinib or dasatinib coadministration on in vitro preantral follicle development and oocyte acquisition in cyclophosphamide-treated mice
}

\author{
Yeon Hee Hong ${ }^{1,2}$, Se Jeong Kim²,3, Seul Ki Kim ${ }^{1,2}$, Seung-Chan Lee ${ }^{4}$, Jin Hyun Jun ${ }^{5}$, Byung Chul Jee ${ }^{1,2}$, Seok Hyun Kim² \\ 'Department of Obstetrics and Gynecology, Seoul National University Bundang Hospital, Seongnam; ${ }^{2}$ Department of Obstetrics and Gynecology, Seoul \\ National University College of Medicine, Seoul; ${ }^{3}$ Department of Obstetrics and Gynecology, Fertility Center of CHA Gangnam Medical Center, CHA \\ University School of Medicine, Seoul; ${ }^{4}$ CNC Biotech Inc., Research Center, Suwon; ${ }^{5}$ Department of Biomedical Laboratory Science, Eulji University, \\ Seongnam, Korea
}

Objective: We investigated the impact of tyrosine kinase inhibitor (imatinib or dasatinib) coadministration with cyclophosphamide (Cp) on preantral follicle development in an in vitro mouse model.

Methods: Seventy-three female BDF1 mice were allocated into four experimental groups: group A, saline; group B, Cp (25 mg/kg); group C, $\mathrm{Cp}(25 \mathrm{mg} / \mathrm{kg})$ and imatinib $(7.5 \mathrm{mg} / \mathrm{kg})$; and group D, Cp (25 mg/kg) and dasatinib $(7.5 \mathrm{mg} / \mathrm{kg})$. Preantral follicles were isolated and cultured in vitro up to 12 days. Final oocyte acquisition and spindle integrity of metaphase II (MII) oocytes were assessed. Levels of $17 \beta$-estradiol and anti-Müllerian hormone (AMH) in the final spent media were measured by enzyme-linked immunosorbent assays, and the mRNA levels of Star, Sod1, Mapk3, and Casp3 in the final follicular cells were quantified by real-time polymerase chain reaction.

Results: The percentage of MII oocytes per initiated follicle, the proportion of MII oocytes with normal spindles, and the 17ß-estradiol level were similar in all four groups. The median AMH level in group B $(7.74 \mathrm{ng} / \mathrm{mL})$ was significantly lower than that in group A $(10.84 \mathrm{ng} / \mathrm{mL})$. However, the median AMH levels in group C $(9.96 \mathrm{ng} / \mathrm{mL})$ and group D $(9.71 \mathrm{ng} / \mathrm{mL})$ were similar to that in group $A$. The mRNA expression levels of Star, Sod1, Mapk3, and Casp3 were similar in all four groups.

Conclusion: Coadministration of imatinib or dasatinib with $\mathrm{Cp}$ could preserve AMH production capacity in this in vitro mice preantral follicle culture model, and it did not affect MII oocyte acquisition.

Keywords: Cyclophosphamide; Dasatinib; Imatinib; Oocyte; Tyrosine kinase

\section{Introduction}

Administration of cyclophosphamide $(\mathrm{Cp})$ to female cancer pa-

Received: April 10, 2020 · Revised: July 20, 2020 - Accepted: September 16, 2020 Corresponding author: Byung Chul Jee

Department of Obstetrics and Gynecology, Seoul National University Bundang Hospital, 82 Gumi-ro 173beon-gil, Bundang-gu, Seongnam 13620, Korea Tel: +82-31-787-7254 Fax: +82-31-787-4054 E-mail: blasto@snubh.org

*This work was supported by grant no. 02-2018-017 from the SNUBH Research fund.

This is an Open Access article distributed under the terms of the Creative Commons Attribution Non-Commercial License (http://creativecommons.org/licenses/by-nc/4.0/) which permits unrestricted non-commercial use, distribution, and reproduction in any medium, provided the original work is properly cited. tients may severely impair ovarian function or lead to premature ovarian failure [1-4]. The mechanisms of Cp-induced ovarian follicular damage are not fully understood, although a proposed mechanism is the apoptotic pathway that occurs in response to intracellular DNA damage [5-7]. Cp-induced intracellular apoptosis frequently occurs in growing follicles in the ovary, especially in proliferating granulosa cells [8]. In response to intracellular DNA damage caused by $\mathrm{Cp}$, intracellular c-Abl is strongly activated, and cell death is subsequently induced through the TAp63 pathway [9-11]. The c-Abl protein is fused to the breakpoint cluster region $(B C R)$ protein to form the breakpoint cluster region-Abelson murine leukemia viral oncogene 1 (BCR-ABL) complex, which acts as an oncogenic tyrosine ki- 
nase, thus leading to cell deformation and leukemic disease.

Tyrosine kinase inhibitors, such as imatinib or dasatinib, are used clinically to treat chronic myeloid leukemia and acute lymphoblastic leukemia. Tyrosine kinase inhibitors inhibit c-Abl or the BCR-ABL complex. The inhibitory effect of dasatinib on BCR-ABL complex is 324 times stronger than that of imatinib, and dasatinib is therefore used in patients with resistance to imatinib [12].

Simultaneous administration of imatinib with cisplatin in mice has been reported to be effective in preserving ovarian follicles $[10,11]$. In those reports, it has been proposed that imatinib could inhibit the c-Abl-TAp63 pathway, thereby reducing cisplatin-induced follicular damage in the mouse ovary. Furthermore, coadministration of imatinib in Cp-treated mice has been reported to increase the oocyte yield, fertilization rate, and the embryo developmental rate [13]. However, there have been no reports regarding the ovoprotective role of dasatinib. In this study, we investigated the impact of imatinib or dasatinib coadministration on in vitro preantral follicle development and oocyte acquisition in Cp-treated mice.

\section{Methods}

\section{In vitro culture of mice preantral follicles}

Female 7- to 8-week-old BDF1 mice (Orient Co., Seoul, Korea) were maintained under 12-hour light and a 12-hour dark conditions at $23^{\circ} \mathrm{C}$ and fed ad libitum. All experiments were conducted ethically with the approval of the Institute of Animal Care and Use Committee of the Seoul National University Bundang Hospital (IACUC No. BA1903-267-014-04).

After 1 week of adaptation, mice were divided into 4 groups and then treated with an intraperitoneal injection of each agent. In group $\mathrm{A}$, which served as a control group, $0.1 \mathrm{~mL}$ of saline was injected once. In group B, Cp ( 25 mg/kg body weight) (Cp monohydrate; Cat. no. 29875, Sigma-Aldrich, St. Louis, MO, USA) was injected once. In group C, Cp (25 mg/kg) and imatinib (7.5 mg/kg; Enzo Life Sciences, Farmingdale, NY, USA) was injected once. In group D, Cp (25 mg/kg) and dasatinib (7.5 mg/kg; Enzo Life Sciences) was injected once. An imatinib or dasatinib stock solution was obtained by dissolving imatinib or dasatinib powder in phosphate-buffered saline (PBS). Three days later, the mice were killed by cervical dislocation, and the bilateral ovaries were collected in $1 \mathrm{~mL}$ of L-15 medium (WelGENE, Dae$\mathrm{gu}$, Korea) supplemented with $0.4 \%$ bovine serum albumin (BSA; Sigma-Aldrich).

Intact preantral follicles were mechanically isolated and cultured in 96-well plates (BD BioCoat; BD Falcon, Franklin Lakes, NJ, USA) at $37^{\circ} \mathrm{C}$ in $5 \% \mathrm{CO}_{2}^{\circ} \mathrm{C}$ for 10 days in a growth medium. The growth medium was composed of alpha-minimum essential medium (WelGENE), $5 \%$ fetal bovine serum (Gibco, Paisley, UK), $10 \mathrm{mlU} / \mathrm{mL}$ recombinant follicle-stimulating hormone (Merck-Serono, Geneva, Switzerland), $1 \%$ insulin-transferrin-selenium mixture (Sigma-Aldrich), and 1\% penicillin-streptomycin mixture (Sigma-Aldrich). Every 3-4 days, the medium was changed, and follicle survival and formation of the antrum were assessed. Oocytes were considered to be dead if they were not surrounded by granulosa cells or if the granulosa cells appeared to be dark and fragmented, and the follicle decreased in size.

After 10 days of culture in the growth medium, the follicles were transferred to maturation medium and cultured for 16 hours at $37^{\circ} \mathrm{C}$ in $5 \% \mathrm{CO}_{2}$. The maturation medium was prepared by adding $1.5 \mathrm{IU} / \mathrm{mL}$ human chorionic gonadotropin (Merck-Serono), and $5 \mathrm{ng} / \mathrm{mL}$ recombinant mouse epidermal growth factor (Sigma-Aldrich) to the growth medium. The oocytes were then harvested either from spontaneously ruptured or non-ruptured follicles. The surrounding cumulus cells were removed by treating them with $0.3 \%$ hyaluronidase (Sigma-Aldrich) and gentle pipetting. Oocytes were classified as metaphase II (MII), metaphase I (MI), germinal vesicle, dead, or degenerated. If a polar body was present in the perivitelline space, the oocytes were regarded as MII oocytes. Fragmented or shrunken oocytes were classified as degenerated oocytes. Figure 1 shows mouse preantral follicles and various developmental stages of follicles during in vitro culture, as well as in the finally obtained oocytes.

\section{Meiotic spindle integrity}

The spindle integrity of the MII oocytes was assessed using previously described methods [14]. The MII oocytes were washed three times with $1 \%$ BSA in PBS for 5 minutes and then fixed with 4\% paraformaldehyde for 1 hour at room temperature (RT). After washing twice with $1 \%$ BSA in PBS, permeabilization was performed with $0.25 \%$ Triton X-100 in PBS for 10 minutes at RT. After washing twice with $1 \% \mathrm{BSA}$ in PBS, blocking was performed with 3\% BSA in PBS for 1 hour at RT and then washing twice with 1\% BSA in PBS. A primary antibody for a-tubulin (Cell Signaling, Danvers, MA, USA) diluted in $1 \%$ BSA (1:100) was added and incubated overnight at $4^{\circ} \mathrm{C}$. After washing three times with $1 \%$ BSA in PBS, a secondary antibody (Alexa Fluor 488 goat anti-rabbit immunoglobulin G; Invitrogen, Carlsbad, CA, USA) diluted in 1\% BSA (1:100) was added for 1 hour at RT in the dark. After washing three times with $1 \%$ BSA in PBS, the oocytes were air-dried on a silane-coated slide (DAKO, Glostrup, Denmark). The slide was counterstained with 4,6'-diamidino-2-phenylindole (DAPI), and examined using a confocal microscope (LSM 710; Carl Zeiss, Oberkochen, Germany). A typical barrel-shaped microtubule structure between both poles with centrally aligned chromosomes was considered normal (Figure 2).

\section{Measurement of hormones in the final spent media}

In each experimental group, the final spent media, in which five 


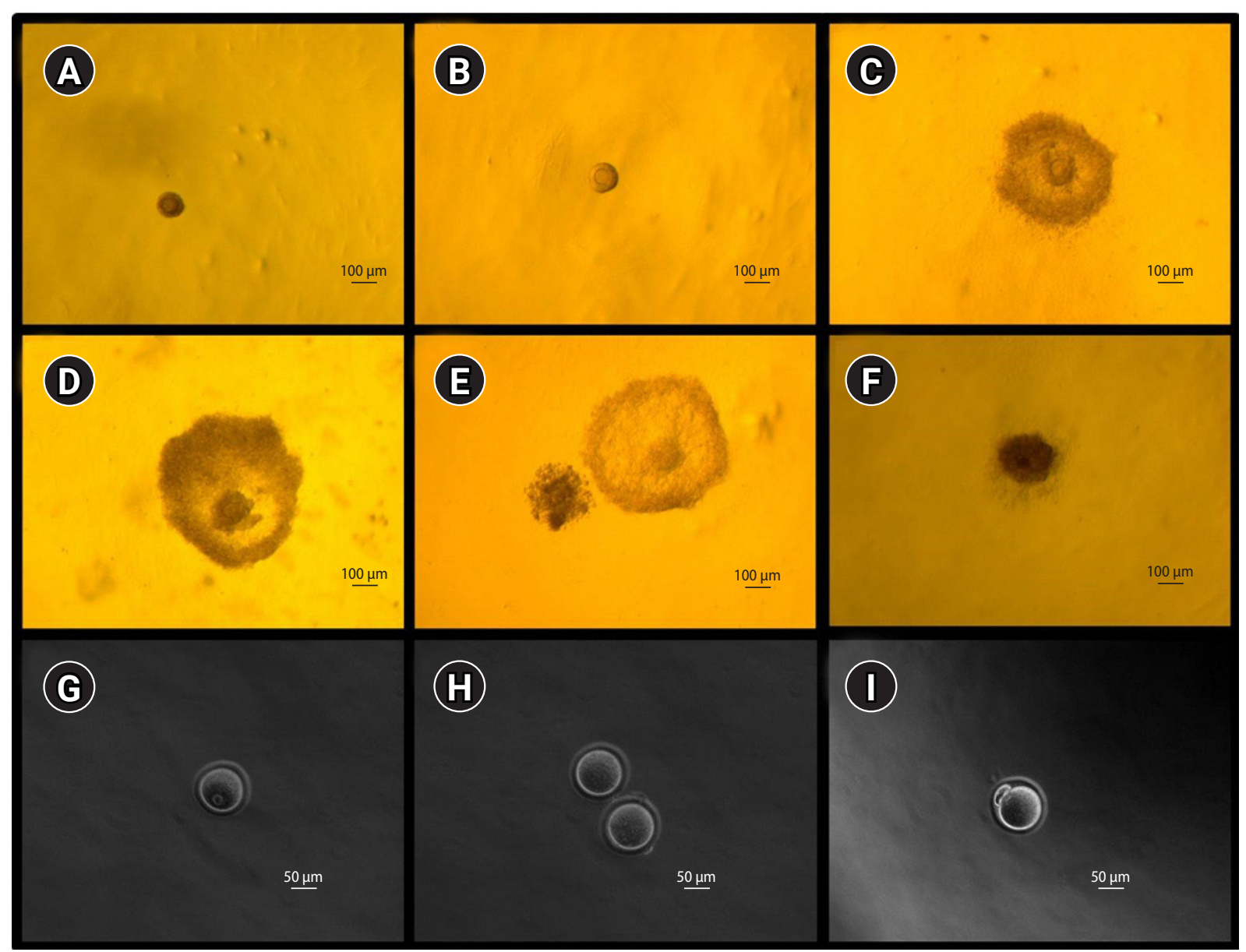

Figure 1. Microphotographs showing mice preantral follicles, in vitro growing follicles (A-F; $\times 60)$ and the resultant oocytes $(G-1 ; \times 200)$. (A) Preantral follicle at day 0, (B) growing follicle at day 4 , (C) growing follicle at day 8 , (D) antral follicle at day 10, (E) ruptured (ovulated) follicle at day 11, (F) dead follicle at day 8, (G) germinal vesicle oocyte, (H) two metaphase I oocytes, and (I) metaphase ll oocyte.

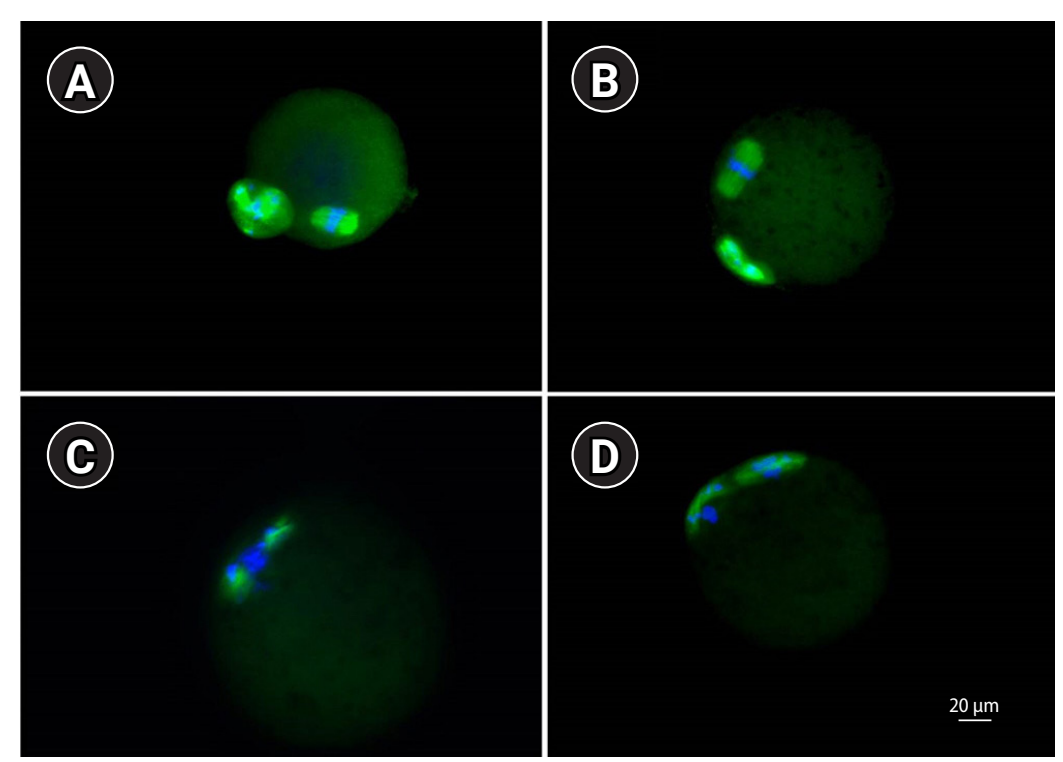

Figure 2. Representative confocal microphotographs showing meiotic spindle organization and chromosome alignment in metaphase II oocytes (×400). (A, B) Normal metaphase II, (C, D) abnormal metaphase II. 
ruptured follicles were present, were pooled and then frozen at $-80^{\circ} \mathrm{C}$. After thawing, the levels of $17 \beta$-estradiol and anti-Müllerian hormone $(\mathrm{AMH})$ were measured by commercially available enzyme-linked immunosorbent assay kits. The limits of sensitivity for $17 \beta$-estradiol (Enzo Life Sciences) and AMH (Ansh Labs, Webster, TX, USA) were $10 \mathrm{pg} / \mathrm{mL}$ and $0.01 \mathrm{ng} / \mathrm{mL}$, respectively.

\section{Real-time quantitative polymerase chain reaction}

The final follicular cells from 20 ruptured follicles were pooled (after isolation of oocytes) and then frozen at $-80^{\circ} \mathrm{C}$. After thawing, total RNA was extracted using the Dynabeads method (Dynabeads mRNA DIRECT kit; Ambion, Oslo, Norway), and cDNAs were synthesized using a PrimeScript first strand cDNA Synthesis Kit (Takara, Kusatsu, Japan) according to the manufacturer's instructions. Real-time quantitative polymerase chain reaction (PCR) was performed using a StepOne-Plus real-time PCR system with TaqMan probes (Applied Biosystems, Foster City, CA, USA). Real-time quantitative PCR was conducted in a $20-\mu \mathrm{L}$ reaction volume containing $10 \mu \mathrm{L}$ of Applied Biosystems TaqMan Universal PCR Master Mix I (Cat. no. 4427788), 2 $\mu \mathrm{L}$ of $\mathrm{cDNA}$, and $6 \mu \mathrm{L}$ of RNase-free water. Specific primers were purchased from Integrated DNA (Coraville, IA, USA). Primer sequences and their product sizes, accession number, annealing temperature, and total PCR cycles of each gene are listed in Table 1.

In this study, the mRNA level of steroidogenic acute regulatory protein (Star; a marker of luteinization), superoxide dismutase 1 (Sod1; a marker of oxidative stress), mitogen-activated protein kinase 3 (Mapk3; a marker of cell growth), and caspase 3 (Casp3; a marker of apoptosis) were quantitatively measured. Glyceraldehyde-3-phosphate dehydrogenase (Gapdh) was used as an internal control. Star transports cholesterol from the outer mitochondrial membrane to the inner membrane and converts it into pregnenolone, which regulates steroid hormone synthesis, especially during the luteal phase [15]. Sod1 is present in the cytoplasm and catalyzes the dispropor- tionation of superoxide into hydrogen peroxide and dioxygen by binding to copper or zinc ions [16]. Mapk3 serves as a signaling molecule and involves cell growth. In the granulosa cells of preovulatory follicles, it is activated by a luteinizing hormone (LH) surge and plays a role in the $\mathrm{LH}$-induced oocyte resumption of meiosis, ovulation, and luteinization [17]. Casp3 is the last enzyme in the cell death process and is a marker of apoptosis $[10,18]$.

In each experimental group, at least five repeats were conducted. Real-time quantitative PCR was repeated five times for each repeat, and the values were averaged. For each experiment, five different readouts were obtained for each gene of interest. The measured values were obtained as the cycle threshold $(\mathrm{Ct})$ at a constant fluorescence intensity. The level of each transcript was inversely related to the observed $\mathrm{Ct}$ value. The relative expression (R) levels of the genes were normalized to that of Gapdh as an internal control. The $\Delta \mathrm{Ct}$ value was calculated as follows: the $\mathrm{Ct}$ of the target gene minus the $\mathrm{Ct}$ of Gapdh. The $\Delta \Delta \mathrm{Ct}$ value was calculated as $\Delta \mathrm{Ct}$ minus the mean value of each group. To determine the fold change for each gene, the relative gene expression of the four treatment groups was calculated using the $2^{-\Delta \Delta c t}$ method as previously described [19].

\section{Statistical analysis}

Statistical analyses were performed using IBM SPSS ver. 22.0 (IBM Corp., Armonk, NY, USA), and $p$-values $<0.05$ were considered to indicate statistical significance. The Fisher exact test was used to compare the proportions among the groups. Numerical data were compared using the Kruskal-Wallis test. If the value was significant, the Mann-Whitney $U$-test with the Bonferroni correction was used for further analysis.

\section{Results}

The detailed outcomes of in vitro growth of preantral follicles and

Table 1. Primer sequences and their conditions for real-time PCR

\begin{tabular}{|c|c|c|c|c|c|}
\hline Gene & Primer sequence & Product size (bp) & Accession number & Annealing temperature $\left({ }^{\circ} \mathrm{C}\right)$ & Cycle \\
\hline \multirow[t]{2}{*}{ Star } & F: CCTCCAAGCGAAACACCTT & 109 & NM_011485 & 60 & 40 \\
\hline & R: GGCATACTCAACAACCAGGAA & & & & \\
\hline Sod1 & R: GGTTCCACGTCCATCAGTATG & & & & \\
\hline \multirow[t]{2}{*}{ Mapk3 } & F:TCCATGAGGTCCTGAACAATG & 112 & NM_011952 & 60 & 40 \\
\hline & R:TCCAGATCTTGCTGCGATTC & & & & \\
\hline Casp3 & R: GACTGATGAGGAGATGGCTTG & & & & \\
\hline \multirow[t]{2}{*}{ Gapdh } & F: GTGGAGTCATACTGGAACATGTAG & 150 & NM_008084 & 60 & 40 \\
\hline & R: AATGGTGAAGGTCGGTGTG & & & & \\
\hline
\end{tabular}

PCR, polymerase chain reaction; Star, steroidogenic acute regulatory protein; Sod1, superoxide dismutase 1; Mapk3, mitogen-activated protein kinase 3; Casp3, caspase 3; Gapdh, glyceraldehyde-3-phosphate dehydrogenase; F, forward; R, reverse. 
the percentage of MIl oocytes with normal spindles in group A (saline), B (Cp), C (Cp and imatinib), and D (Cp and dasatinib) are presented in Table 2. There were no significant differences in the follicle survival rate, antrum formation rate, rupture rate, and total oocyte acquisition rate per initiated follicle among the four groups. The percentage of MII oocytes per initiated follicle and the proportion of MII oocytes with normal spindles were also similar in all four groups.

The median level of $17 \beta$-estradiol in the final spent media was similar among the four groups (Table 3). Nonetheless, the median AMH level in group $B(7.74 \mathrm{ng} / \mathrm{mL})$ was significantly lower than that in group $\mathrm{A}$ (10.84 $\mathrm{ng} / \mathrm{mL})$. However, the median AMH levels in group $C(9.96 \mathrm{ng} / \mathrm{mL})$ and group $D(9.71 \mathrm{ng} / \mathrm{mL})$ were similar to that in group A. The relative mRNA levels of Star, Sod1, Mapk3, and Casp3 in the final follicular cells were similar in all four groups (Figure 3).

\section{Discussion}

We investigated the impact of imatinib or dasatinib coadministration with $\mathrm{Cp}$ on preantral follicle development in an in vitro mouse model. In the groups that received imatinib or dasatinib in addition to $\mathrm{Cp}$, follicle survival, antrum formation, spontaneous follicular rupture, acquisition of total and MII oocytes, and the proportion of MII oocytes with normal spindles were all similar to the Cp-only group. Thus, imatinib or dasatinib coadministration with $\mathrm{Cp}$ might not af- fect in vitro mice preantral follicle development and healthy oocyte acquisition. However, imatinib or dasatinib coadministration with $\mathrm{Cp}$ could preserve AMH levels in the final spent media. This indicates that imatinib or dasatinib coadministration with $\mathrm{Cp}$ could help to preserve AMH production capacity in ruptured follicles in vitro.

In the present study, administration of $\mathrm{Cp}(25 \mathrm{mg} / \mathrm{kg}$ ) reduced $\mathrm{AMH}$ levels but preserved estradiol levels in the final spent media. Generally, AMH is produced principally in primary/preantral follicles, whereas estradiol is mainly produced in antral/ mature follicles. Harvested preantral follicles might be initially damaged by Cp administration, and this damage might reduce $\mathrm{AMH}$ production capacity, which was maintained during the 12-day culture period. However, estradiol is produced mainly in secondary/mature follicles; therefore, in vitro ruptured follicles in the Cp-treated group might produce estradiol to a similar degree to the group that did not receive $\mathrm{Cp}$ treatment.

It has been reported that primordial follicles are most sensitive to Cp treatment, followed by antral and growing follicles [20]. In that report, although growing follicles were sometimes damaged by $\mathrm{Cp}$ treatment, those follicles were found to be the least sensitive to $C p$; they recovered well, and thus could produce estradiol. A further investigation would be necessary to verify the preservation of primor$\mathrm{dial} /$ preantral follicles and their ability to produce AMH after administration of $\mathrm{Cp}$ with imatinib or dasatinib at the ovarian level.

Table 2. Outcomes of in vitro growth of mice preantral follicles under four treatment conditions

\begin{tabular}{|c|c|c|c|c|}
\hline Variable & Saline & $\mathrm{Cp}$ & Cp+imatinib & Cp+dasatinib \\
\hline No. of mice & 15 & 16 & 23 & 19 \\
\hline No. of preantral follicles initiated & 100 & 145 & 143 & 145 \\
\hline No. of follicles survived at day 10 (\% per initiated follicle) & $97(97.0)$ & $131(90.3)$ & $132(92.3)$ & $132(91.0)$ \\
\hline No. of follicles with antrum formation (\% per initiated follicle) & $75(75.0)$ & $91(62.8)$ & $99(69.2)$ & $100(67.0)$ \\
\hline No. of follicles with spontaneous rupture (\% per initiated follicle) & $67(67.0)$ & $84(57.9)$ & $84(58.7)$ & $85(58.6)$ \\
\hline No. of total oocytes (\% per initiated follicle) & $67(67.0)$ & $82(56.6)$ & $83(58.0)$ & $83(57.2)$ \\
\hline No. of degenerated oocytes & 0 & 0 & 0 & 0 \\
\hline No. of GV oocytes & 34 & 44 & 60 & 51 \\
\hline No. of GVBD oocytes & 20 & 28 & 14 & 20 \\
\hline No. of Mll oocytes (\% per initiated follicle) & $13(13.0)$ & $10(6.9)$ & $9(6.3)$ & $12(8.3)$ \\
\hline Proportion of Mll with normal spindle, $\mathrm{n}(\%)$ & $6 / 7(85.7)$ & $6 / 9(66.7)$ & $7 / 9(77.8)$ & $9 / 12(75.0)$ \\
\hline
\end{tabular}

$\mathrm{Cp}$, cyclophosphamide; GV, germinal vesicle; GVBD, germinal vesicle breakdown; MII, metaphase II.

Table 3. Hormone level in the final spent media after in vitro growth of mice preantral follicles under four treatment conditions

\begin{tabular}{|c|c|c|c|c|c|}
\hline Variable & Saline & $\mathrm{Cp}$ & $C p+i m a t i n i b$ & $C p+$ dasatinib & $p$-value ${ }^{\text {a) }}$ \\
\hline Repeat & 18 & 20 & 20 & 20 & \\
\hline $17 \beta$-estradiol (pg/mL) & $366(234-600)$ & $483(191-772)$ & $331(230-612)$ & 481 (160-727) & 0.995 \\
\hline $\mathrm{AMH}(\mathrm{ng} / \mathrm{mL})$ & $10.84^{\mathrm{b})}(9.52-12.54)$ & $7.74^{\mathrm{c})}(6.23-9.06)$ & $9.96(7.97-12.10)$ & $9.71(8.59-12.65)$ & $0.003,<0.001^{b)-c)}$ \\
\hline
\end{tabular}

Values are presented as median (interquartile range).

$\mathrm{Cp}$, cyclophosphamide; $\mathrm{AMH}$, anti-Müllerian hormone.

${ }^{\text {a) }}$ ruskal-Wallis test. 

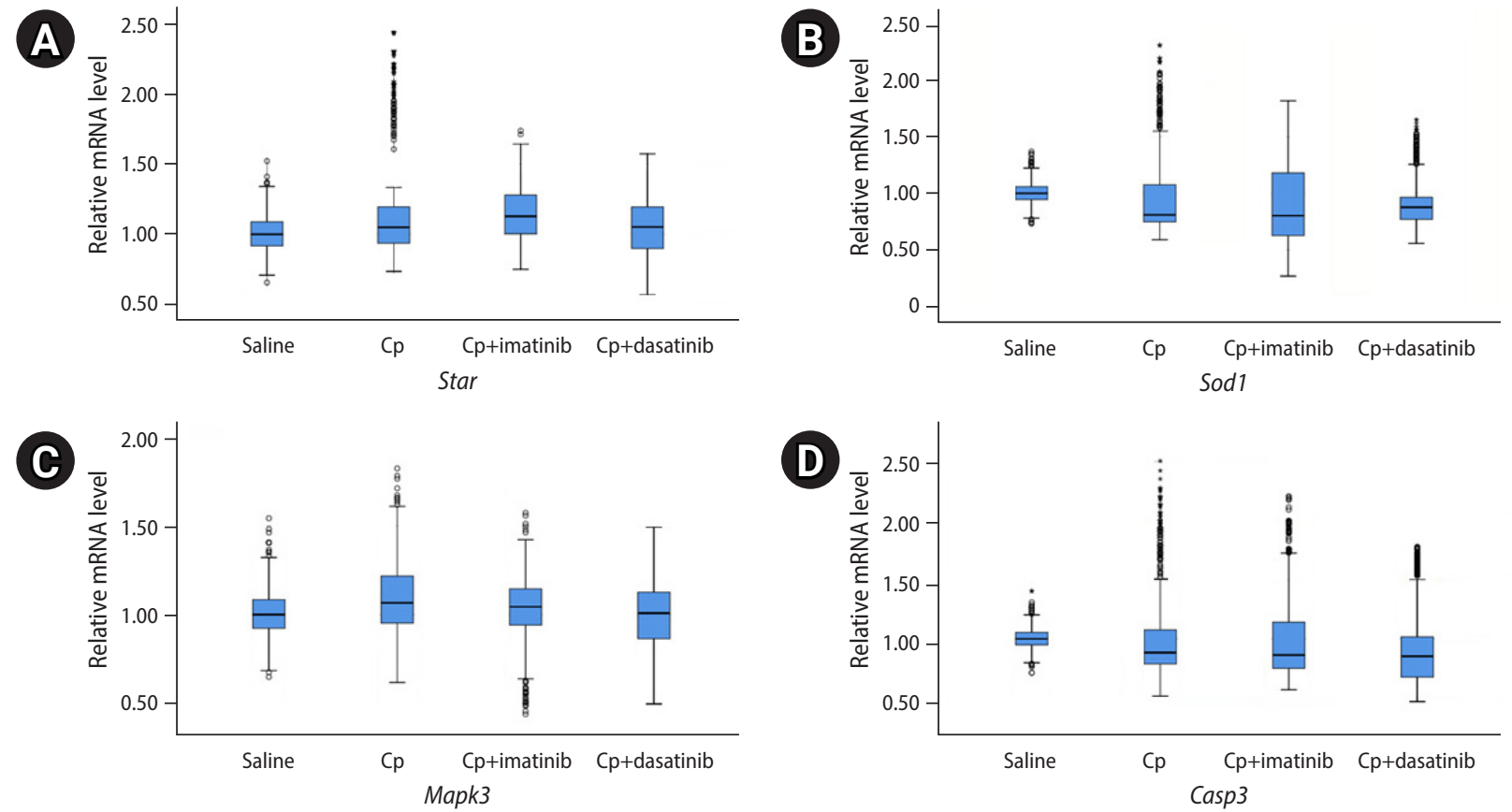

Figure 3. Relative mRNA levels of four genes through real-time polymerase chain reaction. The mRNAs were extracted from the final follicular cells after 12 days of culture of the preantral follicles. The preantral follicles were harvested 3 days after intraperitoneal injections of $0.1 \mathrm{~mL}$ of saline, cyclophosphamide (Cp; 25 mg/kg), Cp (25 mg/kg)+imatinib (7.5 mg/kg), and Cp ( 25 mg/kg)+dasatinib (7.5 mg/kg). (A) Steroidogenic acute regulatory protein (Star), (B) superoxide dismutase 1 (Sod1), (C) mitogen-activated protein kinase 3 (Mapk3), (D) caspase 3 (Casp3).

It is known that AMH suppresses primordial follicle recruitment, thereby maintaining the dormancy of the ovarian follicle pool; in contrast, the loss of AMH activates primordial follicle recruitment and finally depletes the primordial follicle pool [21]. Our finding that $\mathrm{AMH}$ production was preserved in mice that received imatinib or dasatinib coadministration with Cp suggests that imatinib or dasatinib may protect fertility. Imatinib has been reported to play a role in preserving mouse primordial follicles after cisplatin treatment [11,22]. Since imatinib is not a specific inhibitor of tyrosine kinase, dasatinib - as a more specific and potent compound — could play a role as a fertoprotective agent, such as amifostine, ceramide-1-phosphate, or mammalian target of rapamycin inhibitors (e.g., everolimus and rapamycin) $[23,24]$.

A mouse experiment recently demonstrated that imatinib itself has no impact on folliculogenesis. However, it remains generally unknown whether tyrosine kinase inhibitors could modulate the function of ovarian follicles [25]. Since tyrosine kinase inhibitors affect the signaling of c-kit, platelet-derived growth factor receptor, and c-Src, which are also key regulators in the ovary, the direct effect of imatinib or dasatinib on folliculogenesis should be investigated [26].

In the present study, we investigated mouse ovarian follicle development 3 days after exposure to a single dose of imatinib or dasatinib with $\mathrm{Cp}$. However, a further study will be needed to verify the impact of various durations of exposure or various doses of imatinib or dasatinib with $\mathrm{Cp}$. Moreover, further research is required on the impact of imatinib or dasatinib with $\mathrm{Cp}$ on ovarian or embryo-related parameters. In conclusion, coadministration of imatinib or dasatinib with $\mathrm{Cp}$ did not affect in vitro preantral follicle development and healthy oocyte acquisition in mice, but could preserve $\mathrm{AMH}$ production capacity in the final ruptured follicles. Our findings suggest that imatinib and dasatinib might have a fertoprotective role by preserving $\mathrm{AMH}$ production.

\section{Conflict of interest}

Byung Chul Jee has been an editor of Journal of Clinical and Experimental Reproductive Medicine since 2018; however, he was not involved in the peer reviewer selection, evaluation, or decision process of this article. No other potential conflict of interest relevant to this article was reported.

\section{ORCID}

Yeon Hee Hong

Se Jeong Kim

Seul Ki Kim

Seung-Chan Lee

Jin Hyun Jun https://orcid.org/0000-0002-9709-4175 https://orcid.org/0000-0003-1879-3451 https://orcid.org/0000-0002-1647-6711 https://orcid.org/0000-0003-2100-3000 https://orcid.org/0000-0001-9898-4471 
Byung Chul Jee

https://orcid.org/0000-0003-2289-6090

Seok Hyun Kim

https://orcid.org/0000-0003-0649-3224

\section{Author contributions}

Conceptualization: BCJ. Data curation: all authors. Formal analysis: BCJ, YHH, SJK. Funding acquisition: BCJ. Methodology: BCJ, YHH, SJK, SKK, SCL, JHJ. Project administration: BCJ, YHH, SJK, SKK, SCL, JHJ. Writing-original draft: $\mathrm{BCJ}, \mathrm{YHH}$. Writing-review \& editing: all authors.

\section{References}

1. Goswami D, Conway GS. Premature ovarian failure. Hum Reprod Update 2005;11:391-410.

2. Howard-Anderson J, Ganz PA, Bower JE, Stanton AL. Quality of life, fertility concerns, and behavioral health outcomes in younger breast cancer survivors: a systematic review. J Natl Cancer Inst 2012;104:386-405.

3. Letourneau JM, Ebbel EE, Katz PP, Oktay KH, McCulloch CE, Ai WZ, et al. Acute ovarian failure underestimates age-specific reproductive impairment for young women undergoing chemotherapy for cancer. Cancer 2012;118:1933-9.

4. Roness H, Kashi O, Meirow D. Prevention of chemotherapy-induced ovarian damage. Fertil Steril 2016;105:20-9.

5. Meirow D, Nugent D. The effects of radiotherapy and chemotherapy on female reproduction. Hum Reprod Update 2001;7:53543.

6. Oktem O, Oktay K. Quantitative assessment of the impact of chemotherapy on ovarian follicle reserve and stromal function. Cancer 2007;110:2222-9.

7. De Vos M, Devroey P, Fauser BC. Primary ovarian insufficiency. Lancet 2010;376:911-21.

8. Utsunomiya T, Tanaka T, Utsunomiya H, Umesaki N. A novel molecular mechanism for anticancer drug-induced ovarian failure: Irinotecan $\mathrm{HCl}$, an anticancer topoisomerase I inhibitor, induces specific FasL expression in granulosa cells of large ovarian follicles to enhance follicular apoptosis. Int J Oncol 2008;32:991-1000.

9. Livera G, Petre-Lazar B, Guerquin MJ, Trautmann E, Coffigny H, Habert R. p63 null mutation protects mouse oocytes from radio-induced apoptosis. Reproduction 2008;135:3-12.

10. Gonfloni S, Di Tella L, Caldarola S, Cannata SM, Klinger FG, Di Bartolomeo C, et al. Inhibition of the c-Abl-TAp63 pathway protects mouse oocytes from chemotherapy-induced death. Nat Med 2009;15:1179-85.

11. Kim SY, Cordeiro MH, Serna VA, Ebbert K, Butler LM, Sinha S, et al. Rescue of platinum-damaged oocytes from programmed cell death through inactivation of the p53 family signaling network. Cell Death Differ 2013;20:987-97.

12. Lombardo LJ, Lee FY, Chen P, Norris D, Barrish JC, Behnia K, et al. Discovery of N-(2-chloro-6-methyl- phenyl)-2-(6-(4-(2-hydroxyethyl)-piperazin-1-yl)-2-methylpyrimidin-4- ylamino)thiazole-5-carboxamide (BMS-354825), a dual Src/Abl kinase inhibitor with potent antitumor activity in preclinical assays. J Med Chem 2004; 47:6658-61.

13. Chun EK, Jee BC, Kim JY, Kim SH, Moon SY. Effect of imatinib coadministration on in vitro oocyte acquisition and subsequent embryo development in cyclophosphamide-treated mice. Reprod Sci 2014;21:906-14.

14. Kim SK, Jee BC, Kim SH. Effects of supplementation of human endometriotic fluids on in vitro mouse preantral follicle culture. Reprod Sci 2018;25:683-9.

15. Manna PR, Stetson CL, Slominski AT, Pruitt K. Role of the steroidogenic acute regulatory protein in health and disease. Endocrine 2016;51:7-21.

16. Devine PJ, Perreault SD, Luderer U. Roles of reactive oxygen species and antioxidants in ovarian toxicity. Biol Reprod 2012;86:27.

17. Fan HY, Liu Z, Shimada M, Sterneck E, Johnson PF, Hedrick SM, et al. MAPK3/1 (ERK1/2) in ovarian granulosa cells are essential for female fertility. Science 2009;324:938-41.

18. Desmeules P, Devine PJ. Characterizing the ovotoxicity of cyclophosphamide metabolites on cultured mouse ovaries. Toxicol Sci 2006;90:500-9.

19. Shirazi A, Naderi MM, Hassanpour H, Heidari M, Borjian S, Sarvari $A$, et al. The effect of ovine oocyte vitrification on expression of subset of genes involved in epigenetic modifications during oocyte maturation and early embryo development. Theriogenology 2016;86:2136-46.

20. Plowchalk DR, Mattison DR. Reproductive toxicity of cyclophosphamide in the C57BL/6N mouse: 1. effects on ovarian structure and function. Reprod Toxicol 1992;6:411-21.

21. Durlinger AL, Kramer P, Karels B, de Jong FH, Uilenbroek JT, Grootegoed JA, et al. Control of primordial follicle recruitment by anti-Müllerian hormone in the mouse ovary. Endocrinology 1999; 140:5789-96.

22. Morgan S, Lopes F, Gourley C, Anderson RA, Spears N. Cisplatin and doxorubicin induce distinct mechanisms of ovarian follicle loss; imatinib provides selective protection only against cisplatin. PLoS One 2013;8:e70117.

23. Barekati Z, Golkar-Narenji A, Totonchi M, Radpour R, Gourabi H. Effects of amifostine in combination with cyclophosphamide on female reproductive system. Reprod Sci 2012;19:539-46.

24. Pascuali N, Scotti L, Di Pietro M, Oubina G, Bas D, May M, et al. Ceramide-1-phosphate has protective properties against cyclophos- 
phamide-induced ovarian damage in a mice model of premature ovarian failure. Hum Reprod 2018;33:844-59.

25. Schultheis B, Nijmeijer BA, Yin H, Gosden RG, Melo JV. Imatinib mesylate at therapeutic doses has no impact on folliculogenesis or spermatogenesis in a leukaemic mouse model. Leuk Res 2012;
36:271-4.

26. Seeliger MA, Nagar B, Frank F, Cao X, Henderson MN, Kuriyan J. c-Src binds to the cancer drug imatinib with an inactive Abl/c-Kit conformation and a distributed thermodynamic penalty. Structure 2007;15:299-311. 